\title{
Distance Measurements Related to Cartesian Product of Cycles
}

\author{
Xiaoli Qiang, ${ }^{1}$ Saima Nazeer, ${ }^{2}$ Yu-Ming Chu ${ }^{1 D},{ }^{3,4}$ Muhammad Awais Umar $\left(D,{ }^{5}\right.$ \\ Imrana Kousar, ${ }^{2}$ and Ammara Sehar ${ }^{2}$ \\ ${ }^{1}$ Institute of Computing Science and Technology, Guangzhou University, Guangzhou 510006, China \\ ${ }^{2}$ Department of Mathematics, Lahore College For Women University, Lahore 54000, Pakistan \\ ${ }^{3}$ Department of Mathematics, Huzhou University, Huzhou 313000, China \\ ${ }^{4}$ Hunan Provincial Key Laboratory of Mathematical Modeling and Analysis in Engineering, \\ Changsha University of Science \& Technology, Changsha 410114, China \\ ${ }^{5}$ Government Degree College (B), Sharaqpur Sharif, Tehsil 39460, Pakistan
}

Correspondence should be addressed to Yu-Ming Chu; chuyuming@zjhu.edu.cn

Received 2 July 2020; Revised 1 October 2020; Accepted 5 November 2020; Published 23 November 2020

Academic Editor: Ji Gao

Copyright (c) 2020 Xiaoli Qiang et al. This is an open access article distributed under the Creative Commons Attribution License, which permits unrestricted use, distribution, and reproduction in any medium, provided the original work is properly cited.

Graph theory and its wide applications in natural sciences and social sciences open a new era of research. Making the graph of computer networks and analyzing it with aid of graph theory are extensively studied and researched in the literature. An important discussion is based on distance between two nodes in a network which may include closeness of objects, centrality of objects, average path length between objects, and vertex eccentricity. For example, (1) disease transmission networks: closeness and centrality of objects are used to measure vulnerability to particular disease and its infectivity; (2) routing networks: eccentricity of objects is used to find vertices which form the periphery objects of the network. In this manuscript, we have discussed distance measurements including center, periphery, and average eccentricity for the Cartesian product of two cycles. The results are obtained using the definitions of eccentricity, radius, and diameter of a graph, and all possible cases (for different parity of length of cycles) have been proved.

\section{Introduction}

Applications of graph theory to computer science, physics, chemistry, biology, social sciences, and statistics open up a new dimension for researchers [1-5]. One of the attributes is distance and its related measurements in the graph. Weighted distance, topological distance, eccentricity, radius, diameter, metric dimension, indices, etc., are such distancerelated terms and have received much attention of researchers [6-8]. One of the fundamental questions related to distance measurement is community detection and location of their emergency facilitation within the network $[9,10]$. The study of networks such as (1) social networks like Facebook, Twitter, LinkedIn, etc., and (2) biological networks like protein-protein interaction, gene transcription, ecological networks, etc., and statistical inference on these network models have been done extensively in $[11,12]$. In distance-based networks, several vertices can have different closeness as well as exactly the same closeness with respect to a particular facility like hospital, electricity, etc. [13-17]. In this paper, we consider the distance measure, vertex eccentricity, and its associated definitions center and periphery. The indices related to vertex eccentricity are discussed in $[18,19]$. For an undirected graph, Goddard et al. in $[20,21]$ have shown the following:

$$
\operatorname{rad}(M) \leq \operatorname{diam}(M) \leq 2 \operatorname{rad}(M) .
$$

Other proved results are

(1) $\operatorname{rad}\left(K_{m}\right)=\operatorname{diam}\left(K_{m}\right)=1$ for $m \geq 2$

(2) $\operatorname{rad}\left(C_{m}\right)=\operatorname{diam}\left(C_{m}\right)=\lfloor m / 2\rfloor$

(3) $\operatorname{rad}\left(K_{p, m}\right)=\operatorname{diam}\left(K_{p, m}\right)=2, p, m \geq 2$

(4) $\operatorname{rad}\left(P_{m}\right)=\lceil(m-1 / 2)\rceil, \operatorname{diam}\left(P_{m}\right)=m-1$

Diameter of a tree, random graphs, and bridge graphs are determined in $[22,23]$, respectively. 
The eccentricity denoted by ec $(x)$ of a node $x$ in a connected graph $M$ is defined as

$$
\mathrm{ec}(x)=\operatorname{Max}\{d(y, x): y \in V(M)\} .
$$

The radius denoted by $\operatorname{rad}(M)$ of a connected graph $M$ is defined as

$$
\operatorname{rad}(M)=\operatorname{Min}\{\operatorname{ec}(x): x \in V(M)\}
$$

The diameter denoted by $\operatorname{diam}(M)$ of a graph is defined as

$$
\operatorname{diam}(M)=\operatorname{Max}\{\operatorname{ec}(x): x \in V(M)\} .
$$

In [24], authors introduced average eccentricity denoted by $\operatorname{Avg}_{\text {ec }}$ for a graph $M$ with $m$ number of vertices as

$$
\operatorname{Avg}_{\text {ec }}(M)=\frac{1}{m} \sum_{x \in V(M)} \mathrm{ec}_{M}(x) \text {. }
$$

Buckley [25] defined the eccentric set of a nontrivial connected graph and proved the criteria for a nonempty set of positive integers to be an eccentric set of some graphs. Buckley also defined central subgraphs embedding and proved that the central subgraph of a tree is isomorphic to $K_{1}$ or $K_{2}$. Dankelmann and Osaye [26] proved results on average eccentricity, $k$-packing, and $k$-domination in graphs. They gave the bounds for average eccentricity of a connected graph with independence number. Additionally, they defined the other parameters related to eccentricity: weight function, total weight function, eccentric sequence of tree for given diameter, and $k$-star. Dankelmann et al. in [27] prove the bounds for eccentricity and average eccentricity of the graph, subgraph, and its complement and when the graph is replaced by a spanning tree or spanning graph. Yu et al. [28, 29] characterize the extremal unicyclic graphs among other $m$-unicyclic graphs with minimal and second minimal average eccentricity. Ilic [30, 31] discusses the graph transformations which change the eccentricity of a graph. He also solved four conjectures about average eccentricity, clique number, domination, and independent number using the system AutoGraphix.

In a graph $M, x$ is an eccentric vertex to $y$ when ec $(y)=d(x, y), y$ is the central vertex if ec $(y)=\operatorname{rad}(M)$, and $y$ is peripheral if ec $(y)=\operatorname{diam}(M)$. A subgraph of $M$ induced by peripheral vertices is called periphery and denoted by $P(M) . C(M)$ is the subgraph induced by central vertices, also called the center of $M$. When all vertices are central vertices and $C(M)=M$, then $M$ is called self-centered. Self-centered graphs were introduced by Akiyama et al. in [32], and results were proved about graphs $K_{p}, p K_{2}, \cup_{i=1}^{n} K_{p_{i}}$, and $\left\langle V-S_{1}\right\rangle_{G}$. Negami and $\mathrm{Xu}$ [33] prove the existence of the cycle of length 4 or 5 in a self-centered graph of radius 2 , and conversely, if the longest cycle among them for a block vertex has a cycle length 4 , then the block is self-centered and radius is 2 . Halina Bielak and Maciey syslo $[26,34]$ investigated that every graph is not periphery of some graph. In graphs with $\operatorname{rad}(G)=\operatorname{diam}(G)$, the center of the graph becomes the same as the graph itself [35].

The paper is divided into two sections, the first section describes center and periphery for the Cartesian product of two cycles $C_{p}$ and $C_{q}$ for different parity of vertices using definitions of eccentricity, radius, and diameter of a graph with few figures. The second section proves results about average eccentricity of the Cartesian product of two cycles for different parity of number of vertices.

\section{Center and Periphery for Cartesian Product $C_{p} \square C_{q}$}

In this section, we will find results related to center and periphery of the Cartesian product $C_{p} \square C_{q}$ for different choices of $p$ and $q$ using the distance-related definitions eccentricity, radius, and diameter. The graph of $\left(C_{4} \square C_{3}\right)$ is shown in Figure 1.

Definition 1 (see [24]). The Cartesian product of two graphs $M$ and $N$ denoted by $M \square N$ is defined as a graph with the vertex set $V(M) \times V(N)$, where the two vertices $(u, \hat{u})$ and $(v, \hat{v})$ are adjacent if and only if either $u=v$ in $M$ and $u$ is adjacent to $v$ in $N$ or $u$ is adjacent to $v$ in $M$ and $u=v$ in $N$.

The vertex set and edge set of the Cartesian product of graphs are defined as

$$
\begin{aligned}
& V\left(C_{p} \square C_{q}\right)=\left\{x_{(r, s)}: 1 \leq r \leq p, 1 \leq s \leq q\right\}, \\
& \left.E\left(C_{p} \square C_{q}\right)=\left\{x_{(r, s)} x_{(r+1, s)}: 1 \leq r \leq p-1,1 \leq s \leq q\right\} \cup\left\{x_{(r, s)} x_{(r, s+1)}: 1 \leq r \leq p, 1 \leq s \leq q-1\right\}\right\} .
\end{aligned}
$$

Theorem 1. The family of the Cartesian product $C_{p} \square C_{q}$ is self-centered.

Proof. We will prove the result for some choices of $p$ and $q$ as given in the following cases:

Case 1 . When $p \equiv 0(\bmod 2), q \equiv 0(\bmod 2), p \geq q$.

Consider the cycle $\left(x_{(1,1)} x_{(1,2)} x_{(1,3)} \ldots x_{(1, r)} \ldots x_{(1, p)}\right)$ and choose an arbitrary vertex $x_{(1,1)}$ on this cycle:

$$
\begin{aligned}
& d\left(x_{(1,1)}, x_{(1, r)}\right)=r-1, \quad 1 \leq r \leq \frac{p}{2}+1, \\
& d\left(x_{(1,1)}, x_{(1, r)}\right)=\frac{p}{2}-1, \quad r=\frac{p}{2}+2, \\
& d\left(x_{(1,1)}, x_{(1, r)}\right)=1, \quad r=p,
\end{aligned}
$$




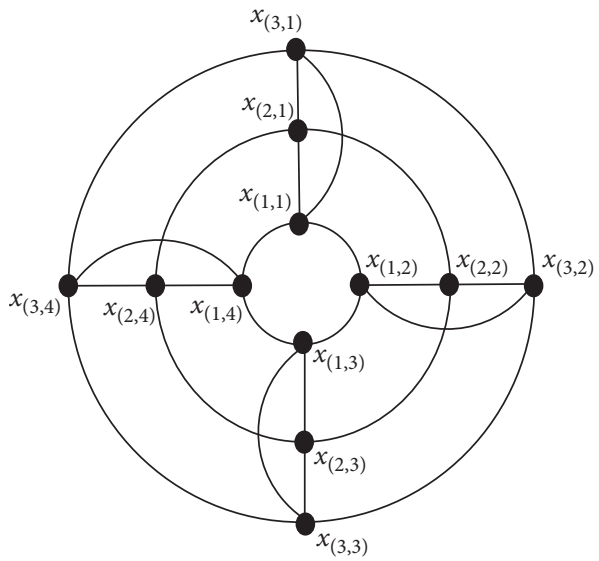

Figure 1: The Cartesian product $\left(C_{4} \square C_{3}\right)$.

also

$$
d\left(x_{(1,1)}, x_{(1, r)}\right)=p+1-r, \quad \frac{p}{2}+2 \leq r \leq p .
$$

In order to locate a vertex at an extreme distance from $x_{(1,1)}$ in $C_{p} \square C_{q}$, we have to consider values $1 \leq r \leq(p / 2)+1$. Since each $x_{(1, r)}$ is the neighbor of $x_{(2, r)}$ and $x_{(q, r)}$, therefore, using equations (7) and (8), we have

$$
d\left(x_{(1,1)}, x_{(q, r)}\right)=d\left(x_{(1,1)}, x_{(2, r)}\right)=r, \quad 1 \leq r \leq \frac{p}{2}+1,
$$

$d\left(x_{(1,1)}, x_{(q, r)}\right)=d\left(x_{(1,1)}, x_{(2, r)}\right)=p+2-r, \quad 2+\frac{p}{2} \leq r \leq p$.

Further, $x_{(2, r)}$ is the neighbor of $x_{(3, r)}$ and $x_{(q, r)}$ is the neighbor of $x_{(q-1, r)}$, and therefore equations (9) and (10) give

$$
\begin{aligned}
& d\left(x_{(1,1)}, x_{(q-1, r)}\right)=d\left(x_{(1,1)}, x_{(3, r)}\right)=r+1, \quad 1 \leq r \leq \frac{p}{2}+1, \\
& d\left(x_{(1,1)}, x_{(q-1, r)}\right)=d\left(x_{(1,1)}, x_{(3, r)}\right)=p+3-r, \quad \frac{p}{2}+2 \leq r \leq p .
\end{aligned}
$$

Moreover, $x_{(3, r)}$ is the neighbor of $x_{(4, r)}$ and $x_{(q-1, r)}$ is the neighbor of $x_{(q-2, r)}$; therefore,

$$
\begin{aligned}
& d\left(x_{(1,1)}, x_{(q-2, r)}\right)=d\left(x_{(1,1)}, x_{(4, r)}\right)=r+2, \quad 1 \leq r \leq \frac{p}{2}+1, \\
& d\left(x_{(1,1)}, x_{(q-2, r)}\right)=d\left(x_{(1,1)}, x_{(4, r)}\right)=p+4-r, \quad \frac{p}{2}+2 \leq r \leq p .
\end{aligned}
$$

Continuing the same procedure, after $(q / 2)$ th steps, the vertex $x_{((q / 2), r)}$ would be in the neighborhood of $x_{((q / 2)+1, r)}$ which implies

$$
\begin{aligned}
& d\left(x_{(1,1)}, x_{(1+(q / 2), r)}\right)=r+\frac{q}{2}-1, \quad 1 \leq r \leq \frac{p}{2}+1, \\
& d\left(x_{(1,1)}, x_{((q / 2)+1, r)}\right)=p-r+\frac{q}{2}+1, \quad \frac{p}{2}+2 \leq r \leq p .
\end{aligned}
$$

This means $x_{((q / 2)+1,(p / 2)+1)}$ is farthest from $x_{(1,1)}$. Therefore, ec $\left(x_{(1,1)}\right)=(p / 2)+(q / 2)$.

Similarly, $\left.x_{((q / 2)+2,(p / 2)+1)}, x_{(}(q / 2)+3,(p / 2)+1\right), x_{((q /}$ $2)+4,(p / 2)+1), \ldots, x_{((q / 2),(p / 2)+1)}$ are farthest from $x_{(2,1)}, x_{(3,1)}, x_{(4,1)}, \ldots, x_{(q, 1)}$ in $C_{p} \square C_{q}$, respectively.

Since the graph is symmetric, each vertex on either cycle has the same eccentricity.

$$
\operatorname{rad}\left(C_{p} \square C_{q}\right)=\operatorname{diam}\left(C_{p} \square C_{q}\right)=\frac{p}{2}+\frac{q}{2} .
$$

Consequently, each vertex is a central vertex as well as a peripheral vertex.

Case 2. When $p \equiv 1, q \equiv 1(\bmod 2), p \geq q$.

Consider the cycle $\left(x_{(1,1)} x_{(1,2)} x_{(1,3)} \ldots x_{(1, r)} \ldots x_{(1, p)}\right)$ and select a vertex $x_{(1,1)}$ from it, and then

$$
d\left(x_{(1,1)}, x_{(1, r)}\right)=r-1, \quad 1 \leq r \leq \frac{p-1}{2}+1 .
$$

When values of $r$ vary between $(p-1 / 2)+2$ and $p$, the distance $d\left(x_{(1,1)}, x_{(1, r)}\right)$ varies between $(p-1 / 2)$ and 1 :

$d\left(x_{(1,1)}, x_{(1, r)}\right)=p+1-r, \quad \frac{p-1}{2}+2 \leq r \leq p$.

Thus, to locate a farthest vertex from $x_{(1,1)}$ in $C_{p} \square C_{q}$, we only consider $1 \leq r \leq(p-1 / 2)+1$.

Each $x_{(1, r)}$ is the neighbor of $x_{(2, r)}$ and $x_{(q, r)}$. Therefore, using equations (15) and (16), we have

$$
d\left(x_{(1,1)}, x_{(q, r)}\right)=d\left(x_{(1,1)}, x_{(2, r)}\right)=r, \quad 1 \leq r \leq \frac{p-1}{2}+1,
$$

$$
\begin{aligned}
d\left(x_{(1,1)}, x_{(q, r)}\right) & =d\left(x_{(1,1)}, x_{(2, r)}\right) \\
& =p+2-r, \quad \frac{p-1}{2}+2 \leq r \leq p .
\end{aligned}
$$


Further, $x_{(2, r)}$ is the neighbor of $x_{(3, r)}$ and $x_{(n, r)}$ is the neighbor of $x_{(q-1, r)}$, and so equations (17) and (18) imply

$$
\begin{aligned}
d\left(x_{(1,1)}, x_{(q-1, r)}\right) & =d\left(x_{(1,1)}, x_{(3, r)}\right) \\
& =1+r, \quad 1 \leq r \leq \frac{p-1}{2}+1, \\
d\left(x_{(1,1)}, x_{(q-1, r)}\right) & =d\left(x_{(1,1)}, x_{(3, r)}\right) \\
& =p+3-r, \quad \frac{p-1}{2}+2 \leq r \leq p .
\end{aligned}
$$

Moreover, $x_{(3, r)}$ is the neighbor of $x_{(4, r)}$ and $x_{(q-1, r)}$ is the neighbor of $x_{(q-2, i)}$. Hence,

$$
\begin{aligned}
d\left(x_{(1,1)}, x_{(q-2, r)}\right) & =d\left(x_{(1,1)}, x_{(4, r)}\right) \\
& =2+r, \quad 1 \leq r \leq \frac{p-1}{2}+1, \\
d\left(x_{(1,1)}, x_{(q-2, r)}\right) & =d\left(x_{(1,1)}, x_{(4, r)}\right) \\
& =p+4-r, \quad \frac{p-1}{2}+2 \leq r \leq p .
\end{aligned}
$$

Continuing the same procedure, after $(q-1 / 2)$ th steps, the vertex $x_{((q-1 / 2), r)}$ would be in the neighbor of $x_{((q+1 / 2), r)}$. Therefore,

$$
\begin{aligned}
& d\left(x_{(1,1)}, x_{((q+1 / 2), r)}\right)=\frac{q-1}{2}+r-1, \quad 1 \leq r \leq \frac{p-1}{2}+1, \\
& d\left(x_{(1,1)}, x_{((q+1 / 2), r)}\right)=p-r+\frac{q-1}{2}+1, \quad \frac{p-1}{2}+2 \leq r \leq p .
\end{aligned}
$$

It means that $x_{((q+1 / 2),(p-1 / 2)+1)}$ is farthest from $x_{(1,1)}$. Therefore, ec $\left(x_{(1,1)}\right)=(p-1 / 2)+(q-1 / 2)$. Similarly, $x_{((q+3 / 2),(p-1 / 2)+1)}, x_{((q+5 / 2),(p-1 / 2)+1)}, x_{((q+7 / 2),}(p-1 / 2)+$ $1), \ldots, x_{((q-1 / 2),(p-1 / 2)+1)}$ are farthest vertices from $x_{(2,1)}, x_{(3,1)}, v_{(4,1)}, \ldots, x_{(q, 1)}$, respectively.

Hence,

$\operatorname{rad}\left(C_{p} \square C_{q}\right)=\operatorname{diam}\left(C_{p} \square C_{q}\right)=(p-1 / 2)+(q-1 / 2)$.

Case 3. Consider $p \equiv 1(\bmod 2), q \equiv 0(\bmod 2), p \geq q$.

The vertices $x_{(1,1)}$ and $x_{(1, r)}$ on the cycle $\left(x_{(1,1)} x_{(1,2)} x_{(1,3)} \ldots x_{(1, r)} \ldots x_{(1, p)}\right)$ have the following distances:

$$
\begin{aligned}
& d\left(x_{(1,1)}, x_{(1, r)}\right)=r-1, \quad 1 \leq r \leq \frac{p-1}{2}+1, \\
& d\left(x_{(1,1)}, x_{(1, r)}\right)=p+1-r, \quad \frac{p-1}{2}+2 \leq r \leq p .
\end{aligned}
$$

This means to locate the farthest vertex from $x_{(1,1)}$ in $C_{p} \square C_{q}$, only these values $1 \leq r \leq(p-1 / 2)+1$ are considered.

Since, each $x_{(1, r)}$ is the neighbor of $x_{(2, r)}$ and $x_{(q, r)}$, therefore using equations (22) and (23), we have

$$
\begin{aligned}
d\left(x_{(1,1)}, x_{(q, r)}\right) & =d\left(x_{(1,1)}, x_{(2, r)}\right)=r, \quad 1 \leq r \leq \frac{p-1}{2}+1, \\
d\left(x_{(1,1)}, x_{(q, r)}\right) & =d\left(x_{(1,1)}, x_{(2, r)}\right) \\
& =p+2-r, \quad \frac{p-1}{2}+2 \leq r \leq p .
\end{aligned}
$$

Further, $x_{(2, r)}$ is the neighbor of $x_{(3, r)}$ and $x_{(q, r)}$ is the neighbor of $x_{(q-1, r)}$. Therefore, equations (24) and (25) give

$$
\begin{aligned}
d\left(x_{(1,1)}, x_{(q-1, r)}\right) & =d\left(x_{(1,1)}, x_{(3, r)}\right) \\
& =r+1, \quad 1 \leq r \leq \frac{p-1}{2}+1, \\
d\left(x_{(1,1)}, x_{(q-1, r)}\right) & =d\left(x_{(1,1)}, x_{(3, r)}\right) \\
& =p+3-r, \quad \frac{p-1}{2}+2 \leq r \leq p .
\end{aligned}
$$

Moreover, $x_{(3, r)}$ is the neighbor of $x_{(4, r)}$ and $x_{(q-1, r)}$ is the neighbor of $x_{(q-2, r)}$. Therefore,

$$
\begin{aligned}
d\left(x_{(1,1)}, x_{(q-2, r)}\right) & =d\left(x_{(1,1)}, x_{(4, r)}\right) \\
& =r+2, \quad 1 \leq r \leq \frac{p-1}{2}+1, \\
d\left(x_{(1,1)}, x_{(q-2, r)}\right) & =d\left(x_{(1,1)}, x_{(4, r)}\right) \\
& =p+4-r, \quad \frac{p-1}{2}+2 \leq r \leq p .
\end{aligned}
$$

Continuing the same procedure, after $(q / 2)$ th steps, the vertex $x_{((q / 2), i)}$ would be in the neighbor of $x_{((q / 2)+1, i)}$ which implies

$$
\begin{aligned}
& d\left(x_{(1,1)}, x_{((q / 2)+1, r)}\right)=r+\frac{q}{2}-1, \quad 1 \leq r \leq \frac{p-1}{2}+1, \\
& d\left(x_{(1,1)}, x_{((q / 2)+1, r)}\right)=p-r+\frac{n}{2}+1, \quad \frac{p-1}{2}+2 \leq r \leq p .
\end{aligned}
$$


It means $x_{((q / 2)+1,(p-1 / 2)+1)}$ is farthest from $x_{(1,1)}$. Therefore, $\operatorname{ec}\left(x_{(1,1)}\right)=(p-1 / 2)+(q / 2)$. Similarly, farthest vertices from $x_{(2, r)}, x_{(3, r)}, \ldots, x_{(q, r)}$ are $x_{((q / 2)+2,(p-1 / 2)+1)}, x_{((n / 2)+3,(p-1 / 2)+1)}, \ldots, x_{((q / 2),(p-1 / 2)+1)}$, respectively. Hence, $\operatorname{rad}\left(C_{p} \square C_{q}\right)=\operatorname{diam}\left(C_{p} \square C_{q}\right)=$ $(p-1 / 2)+(q / 2)$.

Case 4. Consider $p \equiv 0(\bmod 2), q \equiv 1(\bmod 2), p \geq q$.

Since the graph is symmetric, by switching the roles of $p$ and $q$, we get the same case as Case 3 . Therefore, we have discussed all the possible cases.

Now, it is concluded that the family of $C_{p} \square C_{q}$ is selfcentered for all possible values of $p$ and $q$.

2.1. Illustration. Consider the graph $C_{4} \square C_{3}$ shown in Figure 2 in which eccentricity of every vertex is shown by blue circled numbers. Clearly from Figure 2, all vertices have eccentricity 3 . Therefore, the center and periphery for $C_{4} \square C_{3}$ is the graph itself.

\section{Average Eccentricity of $C_{p} \square C_{q}, p \geq q$}

There are $q$ circles in $C_{p} \square C_{q}$ and each has $p$ vertices. Thus, the total number of vertices in $C_{p} \square C_{n}$ is equal to the product of $p$ and $q$. The average eccentricity of $C_{p} \square C_{q}, p \geq q$, will be discussed in the following cases:

Case 1. For $\{p, q\} \equiv 0(\bmod 2)$,

$$
\begin{aligned}
\operatorname{Avg}_{\mathrm{ec}}\left(C_{p} \square C_{q}\right) & =\frac{1}{\mathrm{pq}} \sum_{x \in V(M)} \mathrm{ec}_{M}(x) \\
& =\frac{1}{\mathrm{pq}}\left\{q\left\{p\left(\frac{p+q}{2}\right)\right\}\right\}=\frac{p+q}{2} .
\end{aligned}
$$

Case 2. When $\{p, q\} \equiv 1(\bmod 2)$,

$$
\begin{aligned}
\operatorname{Avg}_{\mathrm{ec}}\left(C_{p} \square C_{q}\right) & =\frac{1}{\mathrm{pq}} \sum_{x \in V(M)} \mathrm{ec}_{M}(x) \\
& =\frac{1}{\mathrm{pq}}\left\{q\left\{p\left(\frac{p+q-2}{2}\right)\right\}=\frac{p+q-2}{2} .\right.
\end{aligned}
$$

Case 3. When $p \equiv 1$ and $q \equiv 0(\bmod 2)$,

$$
\begin{aligned}
\operatorname{Avg}_{\mathrm{ec}}\left(C_{p} \square C_{q}\right) & =\frac{1}{\mathrm{pq}} \sum_{x \in V(m)} \mathrm{ec}_{M}(x) \\
& =\frac{1}{\mathrm{pq}}\left\{q\left\{p\left(\frac{p+q-1}{2}\right)\right\}\right\}=\frac{p+q-1}{2} .
\end{aligned}
$$

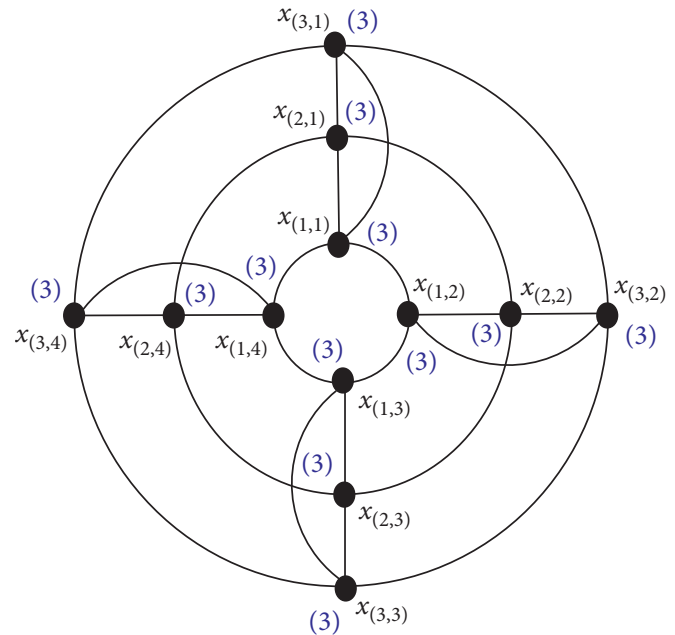

Figure 2: Center and periphery of $\left(C_{4} \square C_{3}\right)$.

Therefore,

$$
\operatorname{Avg}_{\mathrm{ec}}\left(C_{p} \square C_{q}\right)= \begin{cases}\frac{q}{2}+\frac{p}{2}, & \{p, q\} \equiv 0(\bmod 2), \\ \frac{q}{2}+\frac{p-1}{2}, & p \equiv 1 \text { and } q \equiv 0(\bmod 2), \\ \frac{q-1}{2}+\frac{p-1}{2}, & \{p, q\} \equiv 1(\bmod 2) .\end{cases}
$$

Case 4 . When $p \equiv 1$ and $q \equiv 0(\bmod 2)$.

Since the graph is symmetric, by changing the roles of $p$ and $q$, we get Case 3 .

Thus, all cases have been discussed, and our result is completed.

\section{Conclusion}

In this manuscript, we have discussed distance measurements including center, periphery, and average eccentricity for the Cartesian product of two cycles. The results are obtained using the definitions of eccentricity, radius, and diameter of a graph, and all possible cases (for different parity of length of cycles) have been proved. One of the attributes in applications of graph theory is distance and its related measurements in the graph. Weighted distance, topological distance, eccentricity, radius, diameter, metric dimension, indices, etc., are such distance-related terms and have received much attention of researchers. Along with the distance, the graph operations make the structures somewhat similar to the practical situation. One can be interested in researching the distance-related measurements for different graph operations: corona product, strong product, lexicographic product, etc. The other direction might be of 
extending these results for one point union of graphs with nonisomorphic copies.

\section{Data Availability}

All data used for preparation of this manuscript are listed in references.

\section{Conflicts of Interest}

The authors declare that there are no conflicts of interest.

\section{Acknowledgments}

The research was supported by the National Natural Science Foundation of China (Grant nos. 11971142, 11871202, 61673169, 11701176, 11626101, and 11601485).

\section{References}

[1] W. Nazeer, A. Farooq, M. Younas, M. Munir, and S. Kang, "On molecular descriptors of carbon nanocones," Biomolecules, vol. 8, no. 3, p. 92, 2018.

[2] J.-B. Liu, M. Younas, M. Habib, M. Yousaf, and W. Nazeer, "M-Polynomials and Degree-Based Topological Indices of VC5C7 [p, q] and HC5C7 [p, q] Nanotubes," IEEE Access, vol. 7, pp. 41125-41132, 2019.

[3] X. Guo, M. Faheem, Z. Zahid, W. Nazeer, and J. Li, "Faulttolerant resolvability in some classes of line graphs," Mathematical Problems in Engineering, vol. 2020, Article ID 1436872, 8 pages, 2020.

[4] S. M. Kang, M. A. Zahid, A. U. R. Virk, W. Nazeer, and W. Gao, "Calculating the degree-based topological indices of dendrimers," Open Chemistry, vol. 16, no. 1, pp. 681-688, 2018.

[5] Y. C. Kwun, M. Munir, W. Nazeer, S. Rafique, and S. M. Kang, "Computational analysis of topological indices of two boron nanotubes," Scientific Reports, vol. 8, no. 1, pp. 1-14, 2018.

[6] F. Buckley and F. Harary, Distance in Graphs, AddisonWesley, Redwood, CA, USA, 1990.

[7] G. Ranjan and Z. L. Zhang, "On random eccentricity in complex networks," The Tech Report, https://www-users.cs. umn.edu/ ranj0013/Reports/TECHREP_2010_RandEcc.pdf, 2010.

[8] Z. Shi and B. Zhang, "Fast network centrality analysis using GPUs," BMC Bioinformatics, vol. 12, no. 1, p. 149, 2011.

[9] B. Ball, B. Karrer, and M. E. J. Newman, "Efficient and principled method for detecting communities in networks," Physical Review E, vol. 84, no. 3, Article ID 036103, 2011.

[10] A. Chen, A. A. Amini, P. J. Bickel, and E. Levina, "Fitting community models to large sparse networks," 2012, https:// arxiv.org/abs/1207.2340v1.

[11] M. Newman, Networks: An Introduction, OUP, Oxford, UK, 2009.

[12] M. Newman and M. Girvan, "Finding and evaluating community structure in networks," Physical Review E, vol. 69, no. 2, Article ID 026113, 2004.

[13] M. Munir, W. Nazeer, Z. Shahzadi, and S. Kang, "Some invariants of circulant graphs," Symmetry, vol. 8, no. 11, p. 134, 2016.

[14] J.-B. Liu, Z. Zahid, R. Nasir, and W. Nazeer, "Edge version of metric dimension and doubly resolving sets of the necklace graph," Mathematics, vol. 6, no. 11, p. 243, 2018.
[15] Y. C. Kwun, M. Munir, W. Nazeer, S. Rafique, and S. M. Kang, "M-Polynomials and topological indices of V-Phenylenic Nanotubes and Nanotori," Scientific Reports, vol. 7, no. 1, pp. 1-9, 2017.

[16] Y. C. Kwun, A. Ali, W. Nazeer, M. Ahmad Chaudhary, and S. M. Kang, "M-polynomials and degree-based topological indices of triangular, hourglass, and jagged-rectangle benzenoid systems," Journal of Chemistry, vol. 2018, Article ID 8213950, 8 pages, 2018.

[17] Y. Kwun, A. Virk, W. Nazeer, M. Rehman, and S. Kang, "On the multiplicative degree-based topological indices of siliconcarbon Si2C3-I[p,q] and Si2C3-II[p,q]," Symmetry, vol. 10, no. 8 , p. $320,2018$.

[18] G. Yu and L. Feng, "On connective eccentricity index of graphs," MATCH Communications in Mathematical and in Computer Chemistry, vol. 69, p. 611628, 2013.

[19] G. K. Palshikar, "Keyword extraction from a single document using centrality measures," in Proceedings of the International Conference on Pattern Recognition and Machine Intelligence, pp. 503-510, Springer, Berlin, Germany, 2007, December.

[20] W. Goddar and O. R. Oellermann, Distance in graphs, Springer, Berlin, Germany, 2010.

[21] T. W. Valente, K. Coronges, C. Lakon, and E. Costenbader, "How correlated are network centrality measures?" Connections (Toronto, Ont.), vol. 28, no. 1, p. 16, 2008.

[22] G. Chartrand, D. Erwin, and P. Zhang, "Radio antipodal colorings of graphs," Mathematica Bohemica, vol. 127, no. 1, pp. 57-69, 2002.

[23] W. K. Hale, "Frequency assignment: theory and applications," Proceedings of the IEEE, vol. 68, no. 12, pp. 1497-1514, 1980.

[24] G. Chartrand, L. Nebeský, and P. Zhang, "Radio k-colorings of paths," Discussiones Mathematicae Graph Theory, vol. 24, no. 1, pp. 5-21, 2004.

[25] F. Buckley, "Self-centered graphs," Annals of the New York Academy of Sciences, vol. 576, pp. 71-78, 1989.

[26] P. Dankelmann and F. J. Osaye, "Average eccentricity, k-packing and k-domination in graphs," Discrete Mathematics, vol. 342, no. 5, pp. 1261-1274, 2019.

[27] P. Dankelmann, W. Goddard, and C. S. Swart, "The average eccentricity of a graph and its subgraphs," Utilitas Mathematica, vol. 65, pp. 41-52, 2004.

[28] G. Yu, L. Feng, and D. Wang, "On the average eccentricity of unicyclic graphs," Ars Combinatoria, vol. 103, pp. 531-537, 2012.

[29] W. Nazeer, S. Kang, S. Nazeer et al., "On center, periphery and average eccentricity for the convex polytopes," Symmetry, vol. 8, no. 12, p. 145, 2016.

[30] A. Ilic, "On the extremal properties of the average eccentricity," Computers \& Mathematics with Applications, vol. 64, no. 9, pp. 2877-2885, 2012.

[31] K. Das, K. Xu, X. Li, and H. Liu, "On the average of the eccentricities of a graph," Filomat, vol. 32, no. 4, pp. 1395-1401, 2018.

[32] J. Akiyama, K. Ando, and D. Avis, "Eccentric graphs," Discrete Mathematics, vol. 56, no. 1, pp. 1-6, 1985.

[33] S. Negami and G.-H. Xu, "Locally geodesic cycles in 2-self-centered graphs," Discrete Mathematics, vol. 58, no. 3, pp. 263-268, 1986.

[34] D. B. West, Introduction to Graph Theory, Prentice-Hall, Upper Saddle River, NJ, USA, 1996.

[35] G. Chartrand, Introduction to Graph Theory, Tata McGrawHill Education, New York, NY, USA, 2006. 